\title{
Synthesis of novel bisphosphonate polyamine conjugates and their affinity to hydroxyapatite
}

\author{
Elina Sankala*, Janne M. Weisell, Tuulia Huhtala, Ale T. O. Närvänen, \\ and Jouko J. Vepsäläinen \\ School of Pharmacy, Biocenter Kuopio, University of Eastern Finland, \\ P.O. Box 1627, Kuopio, Finland \\ E-mail: elina.sankala@uef.fi
}

Dedicated with respect to Professor Pawel Kafarski

\begin{abstract}
Bisphosphonates are negatively charged molecules, widely used as bone drugs since they have high affinity to bone surface. Polyamines are small organic bases that are positively charged under the physiological conditions and they participate in the regulation of important cellular functions. In this study, we report a general synthesis strategy for bisphosphonate-polyamine conjugates starting from ethenylidene bisphosphonates and $\mathrm{N}$-Boc protected polyamines. Four novel conjugates were prepared and characterized with spectroscopic methods. The affinity of these novel compounds to hydroxyapatite was also determined by using ${ }^{99 \mathrm{~m}} \mathrm{Tc}$ procedure.
\end{abstract}

Keywords: Ethenylidene bisphosphonate, polyamine, synthesis, hydroxyapatite, technetium-99m labelling

\section{Introduction}

Bisphosphonates (BPs) are enzymatically stable negatively charged structural analogs of naturally occurring pyrophosphates (Figure 1). During their lifetime of 50 years, BPs have been used for many purposes starting from water softeners by inhibiting the crystallization of calcium salts and ending up to drug applications. The clinical efficiency of BPs is based on the high affinity for $\mathrm{Ca}^{2+}$ ions in bone mineral hydroxyapatite. ${ }^{1}$ BPs are used in treatment of calcium metabolism disorders, e.g. osteoporosis, because they are effective inhibitors of bone resorption, ${ }^{1}$ and also as bone scanning agents if linked to a gamma-emitting technetium isotope. ${ }^{2}$ 


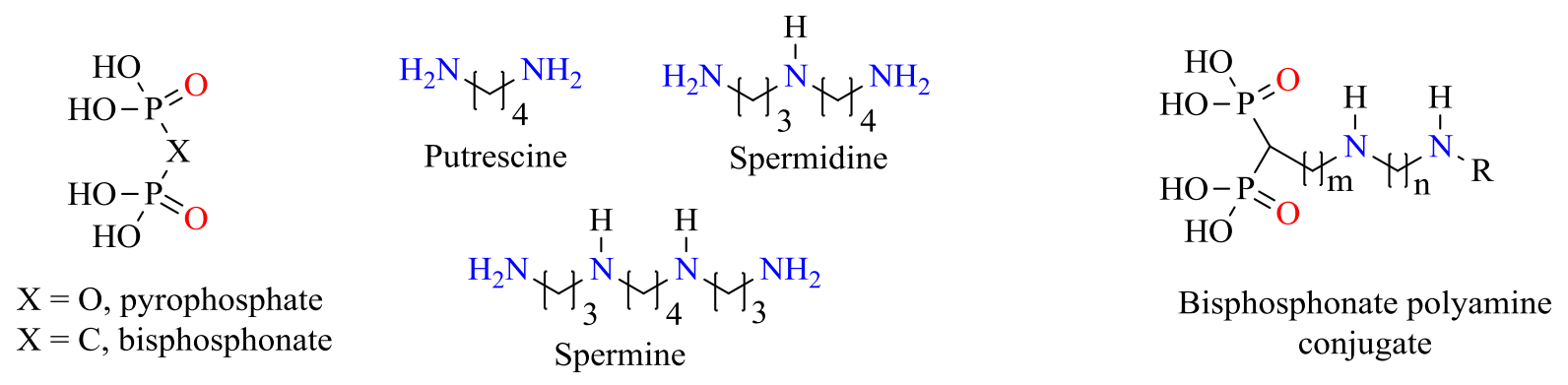

Figure 1. General structure of pyrophosphates, bisphosphonates, natural polyamines and bisphosphonate polyamine conjugates.

Polyamines (PAs) are aliphatic organic bases that are positively charged under the physiological conditions. They exist in millimolar concentrations in all eukaryotic cells and participate in the regulation of important cellular functions, such as proliferation, differentiation and functioning of ion channels like NMDA-receptors and inward rectifier potassium channels. Natural PAs, putrescine, spermidine and spermine (see Figure 1) and their analogues, have been investigated as chemopreventative and antiparasitic agents, NMDA receptor modulators, polyamine-based venoms, metal chelators and as potential carriers for drug delivery. ${ }^{3-5}$ However, while both BPs and PAs are well known compounds there is only some studies related to BP-PA conjugates, in which $\mathrm{m}=\mathrm{n}=2$ and $\mathrm{R}=\mathrm{H}$ (see Figure 1). ${ }^{6,7}$

Here we report the first general synthesis strategy to prepare novel bisphosphonate polyamine (BP-PA) conjugates starting from easily available tetralkyl ethenylidenebisphosphonate. Preparation method allows producing either tetraacidic BPs or partially esterified BPs, which possibly have longer half-life in blood circulation compared to tetraacidic BPs. ${ }^{8}$ The prepared molecules are potential SPECT imaging agents for calcium plaques in brains, since the BP part is an excellent ${ }^{99 \mathrm{~m}}$ Tc chelator and PA moiety is expect to cross the blood-brain-barrier. ${ }^{9,10}$

\section{Results and Discussion}

Synthesis strategies to BP-PA are dependent on the chain length $\mathrm{m}$ (see Figure 1) between the BP bridging carbon and the first nitrogen atom in PA chain. According to literature search the easiest starting point to prepare BP-PA conjugates are ethylidenebisphosphonates, which allow direct Michael addition to activated double bond as shown in Scheme 1, leading to compounds in which $\mathrm{m}=1 .{ }^{11}$ In the case $\mathrm{m}=0$, a three-component reaction is needed between a primary amine, trialkyl orthoformate and dialkyl phosphate, but low yields and unexpected by-products are obtained. ${ }^{12}$ Longer chains $(m>1)$ are typically prepared by adding first appropriately substituted alkyl halide either to $\mathbf{1}$ or $\mathbf{2}$, but additional reaction steps are needed to obtain the target molecule. ${ }^{13}$

Our approach to synthesize these novel BP-PA conjugates (Scheme 1) follows the easiest strategy starting from BP tetraesters $\mathbf{1 a}$ or $\mathbf{b}$, which were first converted into ethenylidene derivatives $\mathbf{2}$ by the known method. ${ }^{11}$ End products $\mathbf{4}$ and $\mathbf{6}$ were obtained without difficulties, 
when $2 \mathbf{a}$ was purified chromatographically ${ }^{14}$ and $\mathbf{2 b}$ was distillated under reduced pressure before use. This strategy also allows to prepare P,P'-diesters of BP-PA conjugates (4c), since selective demethylation of $\mathbf{2 a}$ is obtained with $\mathrm{NaI}$ in acetone to give $\mathbf{2} \mathbf{c}$ in good yield. ${ }^{15}$

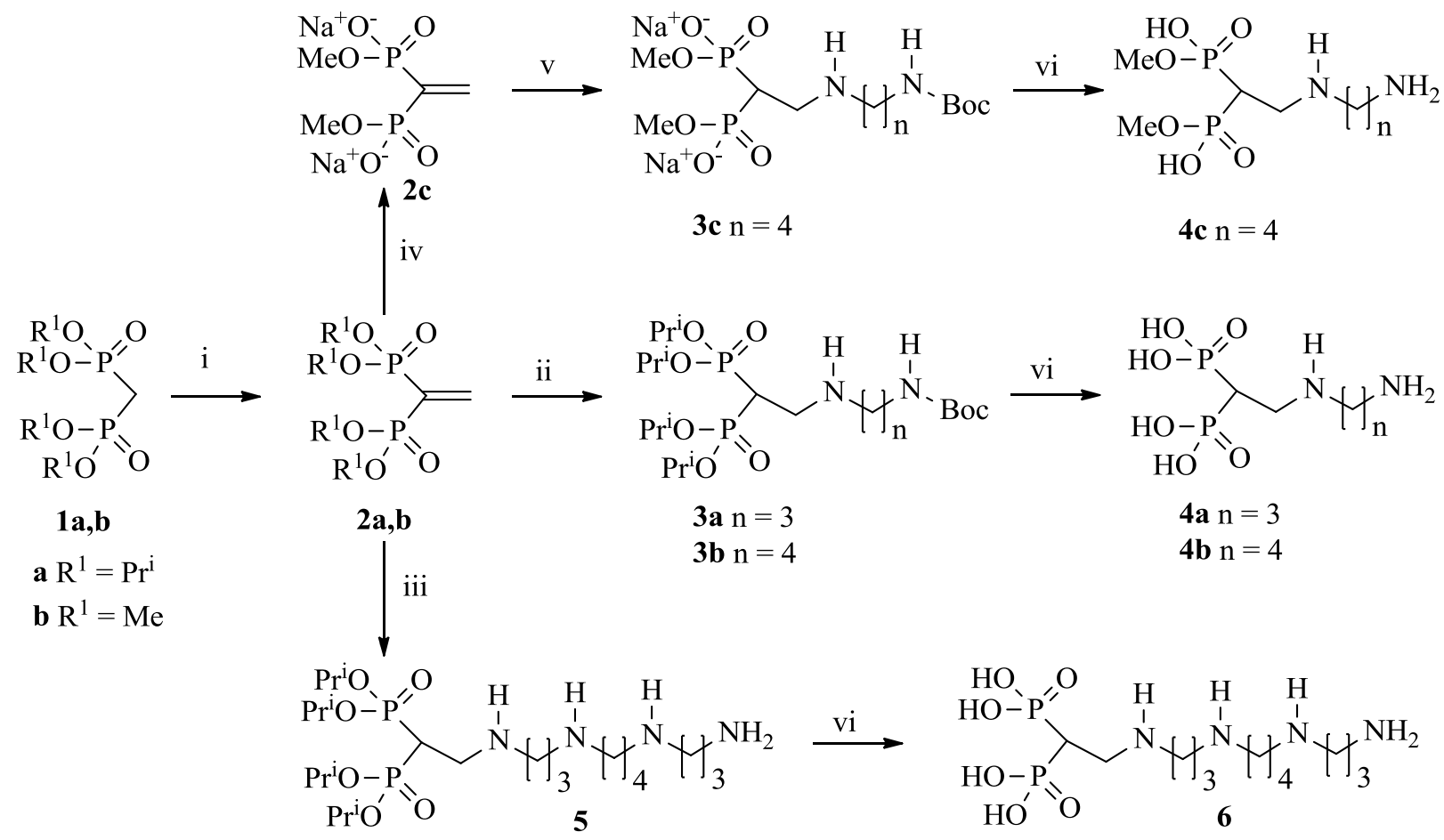

Scheme 1. Synthesis of bisphosphonate-polyamine compounds 6, $4 \mathbf{a}, \mathbf{4 b}$ and $\mathbf{4 c}$. Reagents: (i) 1. $\left(\mathrm{H}_{2} \mathrm{CO}\right)_{\mathrm{n}}, \mathrm{Et}_{2} \mathrm{NH}, \mathrm{MeOH} ; 2$. p-TsOH, toluene; (ii) $\mathrm{H}_{2} \mathrm{~N}\left(\mathrm{CH}_{2}\right)_{\mathrm{n}} \mathrm{NHBoc}$, toluene; (iii)

$\mathrm{H}_{2} \mathrm{~N}\left(\mathrm{CH}_{2}\right)_{3} \mathrm{NH}\left(\mathrm{CH}_{2}\right)_{4} \mathrm{NH}\left(\mathrm{CH}_{2}\right)_{3} \mathrm{NH}_{2}, \mathrm{~K}_{2} \mathrm{CO}_{3}$, toluene; (iv) $\mathrm{NaI}$, acetone; (v) $\mathrm{H}_{2} \mathrm{~N}\left(\mathrm{CH}_{2}\right)_{4} \mathrm{NHBoc}$, methanol; (vi) $4 \mathrm{M} \mathrm{HCl}$.

In the next step two Boc protected PAs ( $N$-Boc-propane-1,3-diamine, $N$-Boc-butane-1,4diamine) were conjugated to $\mathbf{2 a}$ by using Michael type addition. The intermediates $\mathbf{3 a}$ and $\mathbf{3 b}$ were easily obtained after refluxing in toluene, but intermediate $\mathbf{3 c}$ was more complicate to prepare from $\mathrm{P}, \mathrm{P}^{\prime}$-diester 2c due to solubility problems. The best $84 \%$ yield was obtained after 7 days of refluxing in methanol. In the last step both isopropyl and Boc protective groups were simultaneously removed with acid catalysis hydrolysis to give the end products $\mathbf{4 a}$ and $\mathbf{4 b}$. In the case of $\mathbf{4 c}$ only Boc protection group was removed when $\mathbf{3 c}$ was treated with $4 \mathrm{M} \mathrm{HCl}$ and $\mathbf{4 c}$ was obtained with quantitative yield. This method was also tested to prepare more complicated BP-PAs, since $N, N^{\prime}$-bis(3-aminopropyl)butane-1,4-diamine (Spm) afforded without difficulties mostly intermediate $\mathbf{5}$ and according to NMR and mass spectrometry results the only impurity was unreacted Spm starting material.

The prepared compounds were identified by using ${ }^{1} \mathrm{H},{ }^{13} \mathrm{C}$ and ${ }^{31} \mathrm{P}$ NMR spectroscopy and the results are given in the experimental part. Assignments of the signals were verified by using $2 \mathrm{D}{ }^{1} \mathrm{H}$ ${ }^{1} \mathrm{H}$ COSY (homonuclear correlated spectroscopy), 2D ${ }^{13} \mathrm{C}-{ }^{1} \mathrm{H}$ HSQC (heteronuclear single quantum 
correlation spectroscopy), and 2D ${ }^{13} \mathrm{C}-{ }^{1} \mathrm{H}$ HMBC (heteronuclear multiple bond correlation spectroscopy) spectra. Moreover, some interesting spin systems were analyzed by using PERCH software. $^{16}$

In the case of ethenylidene BPs 2, ${ }^{1} \mathrm{H}$ NMR spectra were rather complicated due to $\mathrm{AA}^{\prime} \mathrm{XX}^{\prime}$ spin system in $\mathrm{H}_{2} \mathrm{C}=\mathrm{CP}_{2}$ fragment and four different coupling constants $\left({ }^{2} J_{\mathrm{HH}^{\prime}},{ }^{3} J_{\mathrm{HP}},{ }^{3} J_{\mathrm{HP}^{\prime}}\right.$ and $\left.{ }^{2} J_{\mathrm{PP}^{\prime}}\right)$ were analyzed for $\mathbf{2 a}$ by using PERCH software. Moreover, ${ }^{1} \mathrm{H}$ and ${ }^{13} \mathrm{C}$ NMR spectra for compounds 2a, 3a, 3b, and $\mathbf{5}$ showed two separated $\mathrm{CH}_{3}$ doublets due to the different electronic environments of the $\mathrm{CH}_{3}$ groups. ${ }^{17}$ The same phenomenon was observed also for $\mathrm{OCHMe}_{2}$ carbons. The last peculiarity of the studied compounds was observed in ${ }^{13} \mathrm{C}$ NMR spectra for symmetric ethenylidenes $\mathbf{2 b}$ and $\mathbf{2 c}$. The symmetrical structures of these molecules resulted in an AA'X ( $\mathrm{P}^{\prime}-\mathrm{C}$ $\mathrm{P}-\mathrm{O}-\mathrm{C})$ spin system and $\mathrm{OCH}_{3}$ signal was expected to give a second-order quintet in the carbon spectra. ${ }^{18}$ However, only a triplet was observed due to large ${ }^{2} J_{\mathrm{PP}}$ coupling (ca. $45 \mathrm{~Hz}$ ). Successful addition of PAs to 2 was easily detected from ${ }^{1} \mathrm{H}$ NMR spectra, since characteristic doublet triplet (dt) and triplet triplet (tt) couplings were observed for $\mathrm{N}-\underline{C}_{2}-\underline{C H P}_{2}$ protons, respectively. End products were also characterized with accurate mass spectrometric studies. In some cases (e.g. 4c) sodium adduct, like $[\mathrm{M}+\mathrm{Na}]^{+}$and $\left[\mathrm{M}+\mathrm{Na}_{2}\right]^{+}$give rise to major signal over typical $[\mathrm{M}+\mathrm{H}]^{+}$signal.

The relative binding affinities of the ${ }^{99 \mathrm{~m}} \mathrm{Tc}$ labelled bisphosphonate molecules were studied using hydroxyapatite precipitation tests. ${ }^{19}$ Compounds $\mathbf{4 a - c}$, and $\mathbf{6}$ and etidronate (1-hydroxyethane-1,1-diphosphonic acid) were first labeled with ${ }^{99 \mathrm{~m}} \mathrm{Tc}$. Radiochemical purity was approximately $>99 \%$, with the exception of compound $\mathbf{4 c}$, of which the purity was $90 \%$. This indicates in average high technetium chelating properties of all the compounds. The affinity of the studied compounds with hydroxyapatite (Table 1.) was determined by measuring the remaining activity of the supernatant after centrifugation. ${ }^{19}$ As expected the affinity to the hydroxyapatite was highest with the compound $\mathbf{4 a}$ without any methylation. Also the length of the polyamine spacer affected to the biding efficiency. The affinity of the all polyamine conjugates (4a-c and 6) was higher than the etidronate control. This was expected, as amino-containing bisphosphonates have been shown to have a higher affinity to hydroxyapatite compared to non-amino BPs. ${ }^{20}$

Table 1. The relative affinity of etidronate and BP-PA conjugates to hydroxyapatite after one hour incubation at room temperature. The binding activity of the all BP-PA conjugates was higher than the control compound

\begin{tabular}{cc}
\hline Compound & Affinity to hydroxyapatite (\%) \\
\hline Etidronate & $30 \pm 9$ \\
$\mathbf{4 a}$ & $83 \pm 6$ \\
$\mathbf{4 b}$ & $72 \pm 3$ \\
$\mathbf{4 c}$ & $53 \pm 8$ \\
$\mathbf{6}$ & $72 \pm 19$ \\
\hline
\end{tabular}




\section{Conclusions}

A general method to prepare bisphosphonate polyamine conjugates was developed starting from ethenylidene bisphosphonates and Boc protected polyamines containing one primary amine group. This method allows preparing either tetraacid or partially esterified bisphosphonate polyamine conjugates. Bisphosphonate moieties were labeled with technetium-99m by using conventional stannous chloride reduction under the nitrogen atmosphere. All the conjugates showed radiochemical purity of over $99 \%$ except compound $\mathbf{4 c}(90 \%)$.

\section{Experimental Section}

General. ${ }^{1} \mathrm{H},{ }^{13} \mathrm{C}$, and ${ }^{31} \mathrm{P}$ NMR spectra were recorded at 500.1, 125.8, and 202.4 $\mathrm{MHz}$ on a Bruker Avance 500 DRX (Germany) spectrometer using tetramethylsilane (TMS) in $\mathrm{CDCl}_{3}$ and $\mathrm{MeOD}$ or sodium 3-(trimethylsilyl)-1-propanesulfonate (TSP) in $\mathrm{D}_{2} \mathrm{O}$ as internal standards. High-resolution electrospray mass spectra were obtained on Applied Biosystems/MDS Sciex QSTAR XL. Radioactivity was measured with LKB-Wallac CliniGamma-gamma counter (WALLAC OY, Finland). Tubes for gamma counter and microcentrifuge tubes were purchased from Sarstedt. Centrifuge that was used in the binding assay was Heraeus Biofuge pico. ${ }^{99 \mathrm{~m}}$ Tc-pertechnetate $\left({ }^{99} \mathrm{TcO}_{4}{ }^{-}\right)$in saline solution was obtained as a donation from the Kuopio University Hospital. Thin layer chromatography was accomplished using Merck TLC aluminium sheets (silica gel $60 \mathrm{~F}_{254}$ ) and development was done with potassium permanganate. Column chromatography was carried out on Merck silica gel (70-230 mesh). Instant thin layer chromatography (ITLC) plates were purchased from PALL Life Sciences. Hydroxyapatite powder had been purchased from Sigma. Compounds are named based on ChemOffice name generator.

Tetraisopropyl ethenylidenebisphosphonate (2a). Prepared by the known method ${ }^{11,14}$ from tetraisopropyl methylenediphosphonate. ${ }^{1} \mathrm{H}$ NMR $\left(\mathrm{CDCl}_{3}\right): \delta_{\mathrm{H}} 6.92\left(2 \mathrm{H}, \mathrm{AA}^{\prime} \mathrm{XX}^{\prime}\right.$ spin system, ${ }^{2} J_{\mathrm{HH}^{\prime}}$ $\left.=2.7 \mathrm{~Hz},{ }^{3} J_{\mathrm{HP}}=25.5 \mathrm{~Hz},{ }^{3} J_{\mathrm{HP}^{\prime}}=46.4 \mathrm{~Hz},{ }^{2} J_{\mathrm{PP}^{\prime}}=49.7 \mathrm{~Hz}, \mathrm{CC}_{2}\right), 4.77-4.70\left(4 \mathrm{H}, \mathrm{m}, \mathrm{CHMe}_{2}\right)$, 1.35- $1.31\left(24 \mathrm{H}, \mathrm{m}, \mathrm{CHMe} \underline{2}_{2}\right) .{ }^{13} \mathrm{C} \mathrm{NMR}\left(\mathrm{CDCl}_{3}\right): \delta_{\mathrm{c}} 147.34\left(\underline{\mathrm{CH}}_{2}\right), 134.81\left(\mathrm{t},{ }^{1} J_{\mathrm{CP}}=168.6 \mathrm{~Hz}\right.$, $\left.\mathrm{PCP}), 71.42\left(4 \mathrm{C}, \mathrm{t}^{18},{ }^{2} J_{\mathrm{CP}}=3.0 \mathrm{~Hz}, \mathrm{O} \underline{\mathrm{CH}}\right), 24.04\left(4 \mathrm{C}, \underline{\mathrm{CMe}}_{2}\right), 23.82\left(4 \mathrm{C}, \mathrm{CMe}_{2}\right) .{ }^{31} \mathrm{P} \mathrm{NMR} \mathrm{CDCl}{ }_{3}\right)$ : $\delta_{\mathrm{P}} 12.25$.

Tetramethyl ethenylidenebisphosphonate (2b). Prepared by the known method ${ }^{11}$ from tetramethyl methylenediphosphonate.. ${ }^{1} \mathrm{H}$ NMR $\left(\mathrm{CDCl}_{3}\right): \delta_{\mathrm{H}} 7.13-6.92\left(2 \mathrm{H}, \mathrm{m}, \mathrm{CH}_{2}\right), 3.84-3.78$ $\left(12 \mathrm{H}, \mathrm{m}, \mathrm{OCH}_{3}\right) .{ }^{13} \mathrm{C} \mathrm{NMR}\left(\mathrm{CDCl}_{3}\right): \delta \mathrm{c} 150.09\left(\underline{\mathrm{CH}}_{2}\right), 129.98\left(\mathrm{t},{ }^{1} J_{\mathrm{CP}}=167.5 \mathrm{~Hz}, \mathrm{PCP}\right), 53.09(4 \mathrm{C}$, $\left.\mathrm{t}^{18}, \mathrm{OCH}_{3}\right) .{ }^{31} \mathrm{P} \mathrm{NMR}\left(\mathrm{CDCl}_{3}\right): \delta_{\mathrm{P}} 16.65$.

Dimethyl ethenylidenebisphosphonate disodium salt (2c). Tetramethyl ethenylidenebisphosphonate $2 \mathbf{b}$ (1.0 g, $0.004 \mathrm{~mol}, 1.0$ equiv) and sodium iodide (1.23 g, 0.008 mol, 2.0 equiv) were dissolved in dry acetone $(20 \mathrm{ml})$ and the reaction mixture was kept in $60{ }^{\circ} \mathrm{C}$ for $18 \mathrm{~h}$. The white solid was filtered and washed first with acetone $(20 \mathrm{ml})$ and then with ethyl acetate $(10 \mathrm{ml})$. 
The product was dried under reducer pressure to give disodium salt of 2c (857 $\mathrm{mg}, 82 \%)$ as a white solid. ${ }^{1} \mathrm{H}$ NMR $\left(\mathrm{D}_{2} \mathrm{O}\right): \delta_{\mathrm{H}} 6.61-6.46\left(2 \mathrm{H}, \mathrm{m}, \mathrm{C}_{2}\right), 3.55-3.50\left(6 \mathrm{H}, \mathrm{m}, \mathrm{OC}_{3}\right) .{ }^{13} \mathrm{C} \mathrm{NMR}$ $\left(\mathrm{D}_{2} \mathrm{O}\right): \delta_{\mathrm{c}} 144.96\left(\underline{\mathrm{CH}}_{2}\right), 140.94\left(\mathrm{t},{ }^{1} J_{\mathrm{CP}}=152.4 \mathrm{~Hz}, \mathrm{PCP}\right), 54.69\left(2 \mathrm{C}, \mathrm{t}^{18}, \mathrm{OCH}_{3}\right) .{ }^{31} \mathrm{P}$ NMR $\left(\mathrm{D}_{2} \mathrm{O}\right)$ : $\delta$ Р 14.56.

Tert-butyl [3-[[2,2-bis(diisopropoxyphosphoryl)ethyl]amino]propyl]carbamate (3a). To a stirred suspension of tetraisopropyl ethenylidenebisphosphonate 2 (300 mg, $0.84 \mathrm{mmol}, 1.0$ equiv) in dry toluene $(15 \mathrm{ml})$, was added a solution of $N$-Boc-propane-1,3-diamine (150 mg, $0.84 \mathrm{mmol}$, 1.0 equiv) in dry toluene $(5 \mathrm{ml})$. The reaction mixture was refluxed for $2 \mathrm{~h}$, concentrated under reduced pressure, dissolved in DCM $(10 \mathrm{ml})$, washed with saturated aqueous $\mathrm{NaHCO}_{3}$ solution $(3 \mathrm{x}$ $5 \mathrm{ml})$ and water $(3 \times 5 \mathrm{ml})$, dried $(\mathrm{MgSO} 4)$ and concentrated under reduced pressure to give 3a (350 mg, 78\%) as a yellow oil. ${ }^{1} \mathrm{H}$ NMR $\left(\mathrm{CDCl}_{3}\right): \delta_{\mathrm{H}} 4.85-4.71\left(4 \mathrm{H}, \mathrm{m}, \mathrm{C} \mathrm{HMe}_{2}\right), 3.24-3.16(2 \mathrm{H}$, $\left.\mathrm{m}, \mathrm{CH}_{2} \mathrm{CH}_{2} \mathrm{NH}\right), 3.11\left(2 \mathrm{H}, \mathrm{dt},{ }^{3} J_{\mathrm{HP}}=16.4 \mathrm{~Hz},{ }^{3} J_{\mathrm{HH}}=6.0 \mathrm{~Hz}, \mathrm{CHC}_{2} \mathrm{NH}\right), 2.67-2.61(2 \mathrm{H}, \mathrm{m}$, $\left.\mathrm{NHCH}_{2}\right), 2.49\left(1 \mathrm{H}, \mathrm{tt},{ }^{2} J_{\mathrm{HP}}=23.5 \mathrm{~Hz},{ }^{3} J_{\mathrm{HH}}=6.0 \mathrm{~Hz}, \mathrm{P}_{2} \mathrm{C} \underline{\mathrm{H}}\right), 1.70-1.61\left(2 \mathrm{H}, \mathrm{m}, \mathrm{CH}_{2} \underline{\mathrm{CH}}_{2} \mathrm{CH}_{2}\right), 1.37$ (9H, brs, $\left.\underline{\mathrm{CMe}}_{3}\right), 1.40-1.29\left(24 \mathrm{H}, \mathrm{m}^{17}, \mathrm{CHMe}_{2}\right) .{ }^{13} \mathrm{C} \mathrm{NMR}\left(\mathrm{CDCl}_{3}\right): \delta_{\mathrm{c}} 156.15(\underline{\mathrm{C}}=\mathrm{O}), 71.41(2 \mathrm{C}$, $\left.\left.\mathrm{m}^{17}, \mathrm{OCH}\right), 71.22\left(2 \mathrm{C}, \mathrm{m}^{17}, \mathrm{OCH}\right), 46.86 \mathrm{HNCH}_{2}\right), 46.12\left(\mathrm{t},{ }^{2} J_{\mathrm{CP}}=4.0 \mathrm{~Hz}, \mathrm{CHCH}_{2}\right), 39.19$ $\left(\underline{\mathrm{CH}}_{2} \mathrm{NH}\right), 38.74\left(\mathrm{t},{ }^{1} J_{\mathrm{CP}}=133.7 \mathrm{~Hz}, \mathrm{PCP}\right), 29.74\left(\mathrm{CH}_{2} \underline{\mathrm{CH}}_{2} \mathrm{CH}_{2}\right), 28.55\left(3 \mathrm{C}, \mathrm{CMe}_{3}\right), 24.28(4 \mathrm{C}$, $\left.\mathrm{m}^{17}, \mathrm{CHMe} 2\right), 24.03\left(4 \mathrm{C}, \mathrm{m}^{17}, \mathrm{CHMe}_{2}\right) .{ }^{31} \mathrm{P} \mathrm{NMR}\left(\mathrm{CDCl}_{3}\right): \delta_{\text {Р }} 21.81$.

(2-((3-Aminopropyl)amino)ethane-1,1-diyl)diphosphonic acid (4a). Intermediate 3a was dissolved in $4 \mathrm{M} \mathrm{HCl}(20 \mathrm{ml})$ and the mixture was refluxed for $2 \mathrm{~h}$ and concentrated under reduced pressure. Crystallization from water with ethanol gave $4 \mathbf{a}(156 \mathrm{mg}, 71 \%)$ as a white solid. ${ }^{1} \mathrm{H}$ NMR $\left(\mathrm{D}_{2} \mathrm{O}\right): \delta_{\mathrm{H}} 3.41\left(2 \mathrm{H}, \mathrm{dt},{ }^{3} J_{\mathrm{HP}}=14.2 \mathrm{~Hz},{ }^{3} J_{\mathrm{HH}}=7.3 \mathrm{~Hz}, \mathrm{CHCH}_{2} \mathrm{NH}\right), 3.21-3.14\left(2 \mathrm{H}, \mathrm{m}, \mathrm{NHCH}_{2}\right)$, 3.10-3.02 (2H, m, $\left.\underline{\mathrm{CH}}_{2} \mathrm{NH}_{2}\right), 2.37\left(1 \mathrm{H}, \mathrm{tt},{ }^{2} J_{\mathrm{HP}}=21.0 \mathrm{~Hz},{ }^{3} J_{\mathrm{HH}}=7.3 \mathrm{~Hz}, \mathrm{P}_{2} \mathrm{C} \underline{\mathrm{H}}\right), 2.11-2.02(2 \mathrm{H}, \mathrm{m}$, $\left.\mathrm{CH}_{2} \underline{\mathrm{CH}}_{2} \mathrm{CH}_{2}\right) .{ }^{13} \mathrm{C} \mathrm{NMR}\left(\mathrm{D}_{2} \mathrm{O}\right): \delta \mathrm{c} 45.75\left(\mathrm{CHCH}_{2}\right), 44.61\left(\mathrm{NHCH}_{2}\right), 36.90\left(\underline{\mathrm{CH}}_{2} \mathrm{NH}\right), 36.80\left({ }^{1} J_{\mathrm{CP}}=\right.$ $118.4 \mathrm{~Hz}, \mathrm{PCP}), 24.12\left(\underline{\mathrm{CH}}_{2}\right) .{ }^{31} \mathrm{P} \mathrm{NMR}\left(\mathrm{D}_{2} \mathrm{O}\right): \delta \mathrm{P} 16.12$. HRMS $[\mathrm{M}+\mathrm{H}]^{+}$calculated for $\mathrm{P}_{2} \mathrm{O}_{6} \mathrm{C}_{5} \mathrm{H}_{17} \mathrm{~N}_{2}$ : 263.0562. Found: 263.0564 .

Tert-butyl (4-((2,2-bis(diisopropoxyphosphoryl)ethyl)amino)butyl)carbamate (3b). Preperad as 3a from 2 (500 mg, $1.4 \mathrm{mmol}, 1.1$ equiv) to give 3b (529 mg, 81\%) as a yellow oil. ${ }^{1} \mathrm{H} \mathrm{NMR}$ $\left(\mathrm{CDCl}_{3}\right): \delta_{\mathrm{H}}$ 4.87-4.70 (4H, m, $\left.\mathrm{CHMe}_{2}\right), 3.19-3.01\left(4 \mathrm{H}, \mathrm{m}, \mathrm{CHC}_{2} \mathrm{NH}_{2} \mathrm{CH}_{2} \mathrm{CH}_{2} \mathrm{NH}\right), 2.62-2.56$ $\left(2 \mathrm{H}, \mathrm{m}, \mathrm{NHCH}_{2}\right), 2.49\left(1 \mathrm{H}, \mathrm{tt},{ }^{2} J_{\mathrm{HP}}=23.5 \mathrm{~Hz},{ }^{3} J_{\mathrm{HH}}=6.0 \mathrm{~Hz}, \mathrm{P}_{2} \mathrm{C} \underline{\mathrm{H}}\right), 1.57-1.47(4 \mathrm{H}, \mathrm{m}$, $\mathrm{CH}_{2} \underline{\mathrm{CH}}_{2} \underline{\mathrm{CH}}_{2} \mathrm{CH}_{2}$ ), 1.44 (9H, brs, $\left.\underline{\mathrm{CMe}}_{3}\right), 1.39-1.30\left(24 \mathrm{H}, \mathrm{m}^{17}, \mathrm{CHMe}{ }_{2}\right) .{ }^{13} \mathrm{C} \mathrm{NMR}\left(\mathrm{CDCl}_{3}\right): \delta_{\mathrm{c}}$ $156.12(\underline{\mathrm{C}}=\mathrm{O}), 79.03\left(\underline{\mathrm{CMe}}_{3}\right), 71.41\left(2 \mathrm{C}, \mathrm{m}^{17}, \mathrm{OC} H\right), 71.20\left(2 \mathrm{C}, \mathrm{m}^{17}, \mathrm{OC} H\right), 48.81\left(\mathrm{NHCH}_{2}\right)$, $46.12\left(\mathrm{t},{ }^{2} J_{\mathrm{CP}}=3.9 \mathrm{~Hz}, \mathrm{CHCH}_{2}\right), 40.62\left(\underline{\mathrm{CH}}_{2} \mathrm{NH}\right), 38.94\left(\mathrm{t},{ }^{1} J_{\mathrm{CP}}=133.7 \mathrm{~Hz}, \mathrm{PCP}\right), 28.57(3 \mathrm{C}$, $\left.\mathrm{CMe}_{3}\right), 27.97\left(\underline{\mathrm{CH}}_{2}\right), 27.39\left(\underline{\mathrm{CH}}_{2}\right), 24.33\left(4 \mathrm{C}, \mathrm{m}^{17}, \mathrm{CHMe} 2\right), 24.07\left(4 \mathrm{C}, \mathrm{m}^{17}, \mathrm{CHMe} 2\right) .{ }^{31} \mathrm{P} \mathrm{NMR}$ $\left(\mathrm{CDCl}_{3}\right): \delta$ Р 21.94 .

(2-((4-Aminobutyl)amino)ethane-1,1-diyl)diphosphonic acid (4b). Preparad as 4a from intermediate $\mathbf{3 b}(529 \mathrm{mg}, 0.97 \mathrm{mmol})$ to give $\mathbf{4 b}(253 \mathrm{mg}, 76 \%)$ as a white solid. ${ }^{1} \mathrm{H}$ NMR $\left(\mathrm{D}_{2} \mathrm{O}\right)$ : $\delta_{\text {н }} 3.42\left(2 \mathrm{H}, \mathrm{dt},{ }^{3} J_{\mathrm{HP}}=14.1 \mathrm{~Hz},{ }^{3} J_{\mathrm{HH}}=7.3 \mathrm{~Hz}, \mathrm{CHC}_{2} \mathrm{NH}\right), 3.17-3.08\left(2 \mathrm{H}, \mathrm{m}, \mathrm{NHC}_{2}\right), 3.05-2.97$ $\left(2 \mathrm{H}, \mathrm{m}, \mathrm{CH}_{2} \mathrm{NH}_{2}\right), 2.45\left(1 \mathrm{H}, \mathrm{tt},{ }^{2} J_{\mathrm{HP}}=21.44 \mathrm{~Hz},{ }^{3} J_{\mathrm{HH}}=7.3 \mathrm{~Hz}, \mathrm{P}_{2} \mathrm{CH}\right), 1.82-1.67(4 \mathrm{H}, \mathrm{m}$, $\left.\mathrm{CH}_{2} \underline{\mathrm{C}}_{2} \underline{\mathrm{C}}_{2} \mathrm{CH}_{2}\right) .{ }^{13} \mathrm{C} \mathrm{NMR}\left(\mathrm{D}_{2} \mathrm{O}\right): \delta_{\mathrm{c}} 47.13(\mathrm{NH} \underline{\mathrm{H}} 2), 45.39\left(\mathrm{CHCH}_{2}\right), 39.19\left(\underline{\mathrm{CH}}_{2} \mathrm{NH}\right), 36.61$ (t, 
$\left.{ }^{1} J_{\mathrm{CP}}=120.0 \mathrm{~Hz}, \mathrm{PCP}\right), 24.22\left(\underline{\mathrm{CH}}_{2}\right), 23.10\left(\underline{\mathrm{CH}}_{2}\right) .{ }^{31} \mathrm{P}$ NMR $\left(\mathrm{D}_{2} \mathrm{O}\right): \delta$ P $16.65 . \mathrm{HRMS}[\mathrm{M}+\mathrm{H}]^{+}$ calculated for $\mathrm{P}_{2} \mathrm{O}_{6} \mathrm{C}_{6} \mathrm{H}_{19} \mathrm{~N}_{2}$ : 277.0718. Found: 277.0718 .

Tert-butyl (4-((2,2-bis(hydroxy(methoxy)phosphoryl)ethyl)amino)butyl)carbamate (3c). P,P'dimethyl ester 2c (200 mg, $0.763 \mathrm{mmol}, 1.0$ equiv) and $N$-Boc-butane-1,4-diamine (222 mg, 1.186 mmol, 1.6 equiv) were dissolved in methanol and refluxed for seven days. The reaction mixture was concentrated under reduced pressure, dissolved in water $(10 \mathrm{ml})$, washed with DCM (3 x $5 \mathrm{ml})$, and concentrated again under reduced pressure to give $3 \mathbf{c}(258 \mathrm{mg}, 84 \%)$ as a white solid. ${ }^{1} \mathrm{H}$ NMR (500.1 MHz, MeOD): $\delta$ н $3.61\left(6 \mathrm{H}, \mathrm{d},{ }^{3} J_{\mathrm{HP}}=10.4 \mathrm{~Hz}, \mathrm{OC}_{3}\right), 3.11-2.97\left(4 \mathrm{H}, \mathrm{m}, \mathrm{C}_{2} \mathrm{NH}\right), 2.66-2.55$ $\left(2 \mathrm{H}, \mathrm{m}, \mathrm{NHCH}_{2}\right), 2.33\left(1 \mathrm{H}, \mathrm{tt},{ }^{2} J_{\mathrm{HP}}=21.3 \mathrm{~Hz},{ }^{3} J_{\mathrm{HH}}=6.5 \mathrm{~Hz}, \mathrm{P}_{2} \mathrm{C} \underline{\mathrm{H}}\right), 1.59-1.47\left(4 \mathrm{H}, \mathrm{m}, \mathrm{C}_{2} \mathrm{C}_{2}\right)$, $1.43\left(9 \mathrm{H}, \mathrm{s}, \mathrm{CMe}_{3}\right) .{ }^{13} \mathrm{C}$ NMR $(125.8 \mathrm{MHz}, \mathrm{MeOD}): \delta \mathrm{c} 157.85(\underline{\mathrm{C}}=\mathrm{O}), 79.14\left(\underline{\mathrm{CMe}}_{3}\right), 51.46(2 \mathrm{C}$, $\left.\mathrm{OCH}_{3}\right), 49.05\left(\mathrm{NHCH}_{2}\right), 47.55\left(\mathrm{t},{ }^{2} J_{\mathrm{CP}}=3.0 \mathrm{~Hz}, \mathrm{CHCH}_{2}\right), 40.58\left(\underline{\mathrm{CH}}_{2} \mathrm{NH}_{2}\right), 36.79\left(\mathrm{t},{ }^{1} J_{\mathrm{CP}}=122.6\right.$ $\mathrm{Hz}, \mathrm{P} \underline{\mathrm{CP}}$ ), 28.21 (3C, $\left.\underline{\mathrm{CMe}}_{3}\right), 26.99$ (2C, $\left.\underline{\mathrm{CH}}_{2} \underline{\mathrm{CH}}_{2}\right) .{ }^{31} \mathrm{P}$ NMR (202.4 MHz, MeOD): $\delta$ р 22.01.

Dimethyl (2-((4-aminobutyl)amino)ethane-1,1-diyl)bis(hydrogen phosphonate) (4c) To remove the protective groups $3 \mathbf{c}(100 \mathrm{mg}, 0.3 \mathrm{mmol})$ was dissolved in $4 \mathrm{M} \mathrm{HCl}(5 \mathrm{ml})$ and the mixture was stirred in room temperature for $45 \mathrm{~min}$ and concentrated under reduced pressure to give $\mathbf{4 c}$ ( $96 \mathrm{mg}$, $100 \%)$ as a white solid ${ }^{1} \mathrm{H}$ NMR (MeOD): $\delta_{\mathrm{H}} 3.68\left(6 \mathrm{H}, \mathrm{d},{ }^{3} J_{\mathrm{HP}}=10.7 \mathrm{~Hz}, \mathrm{OCH}_{3}\right), 3.45(2 \mathrm{H}, \mathrm{dt}$, $\left.{ }^{3} J_{\mathrm{HH}}=7.0 \mathrm{~Hz},{ }^{3} J_{\mathrm{HP}}=14.5 \mathrm{~Hz}, \mathrm{CHC}_{2}\right), 3.22-3.12\left(2 \mathrm{H}, \mathrm{m}, \mathrm{NHCH}_{2}\right), 3.10-3.01\left(2 \mathrm{H}, \mathrm{m}, \mathrm{CH}_{2} \mathrm{NH}_{2}\right)$, $2.67\left(1 \mathrm{H}, \mathrm{tt},{ }^{2} J_{\mathrm{HP}}=21.9 \mathrm{~Hz},{ }^{3} J_{\mathrm{HH}}=7.0 \mathrm{~Hz}, \mathrm{P}_{2} \mathrm{CH}\right), 1.87-1.72\left(4 \mathrm{H}, \mathrm{m}, \mathrm{C}_{2}\right) .{ }^{13} \mathrm{C} \mathrm{NMR}(\mathrm{MeOD}): \delta_{\mathrm{c}}$ $55.30\left(2 \mathrm{C}, \mathrm{OCH}_{3}\right), 49.88\left(\mathrm{NHCH}_{2}\right), 47.48\left(\mathrm{CHCH}_{2}\right), 41.70\left(\underline{\mathrm{CH}}_{2} \mathrm{NH}_{2}\right), 36.59\left(\mathrm{t},{ }^{1} J_{\mathrm{CP}}=125.0 \mathrm{~Hz}\right.$, PCP), $26.71\left(\mathrm{CH}_{2} \underline{C H}_{2}\right), 25.52\left(\underline{C H}_{2} \mathrm{CH}_{2}\right) .{ }^{31} \mathrm{P}$ NMR (MeOD): $\delta$ P 19.38. HRMS [M+H] ${ }^{+}$calculated for $\mathrm{P}_{2} \mathrm{O}_{6} \mathrm{C}_{8} \mathrm{H}_{23} \mathrm{~N}_{2}$ : 305.1031. Found: 305.1031 .

Tetraisopropyl (2-((3-((4-((3-aminopropyl)amino)butyl)amino)propyl)amino)ethane-1,1diyl)bis(phosphonate) (5) To a stirred suspension of tetraisopropyl ethenylidenebisphosphonate $\mathbf{2}$ (300 mg, $0.84 \mathrm{mmol}, 1.0$ equiv) in dry toluene $(15 \mathrm{ml})$, was added a solution of $N, N^{\prime}-\operatorname{di}(3-$ aminopropyl)butane-1,4-diamine $(170 \mathrm{mg}, 0.84 \mathrm{mmol}, 1.0$ equiv) in dry toluene $(5 \mathrm{ml})$. The reaction mixture was refluxed for $2 \mathrm{~h}$, filtered, and concentrated under reduced pressure to give 5 (390 mg, 83\%, purity ca. $80 \%)$ as a pale yellow oil. ${ }^{1} \mathrm{H} \mathrm{NMR}\left(\mathrm{CDCl}_{3}\right): \delta_{\mathrm{H}} 4.84-4.71(4 \mathrm{H}, \mathrm{m}$, $\left.\mathrm{C}_{\mathrm{HMe}}\right), 3.11\left(2 \mathrm{H}, \mathrm{dt},{ }^{3} J_{\mathrm{HP}}=16.4 \mathrm{~Hz},{ }^{3} \mathrm{~J}_{\mathrm{HH}}=5.9 \mathrm{~Hz}, \mathrm{P}_{2} \mathrm{CHC}_{2}\right), 2.79-2.74(2 \mathrm{H}, \mathrm{m}$, $\left.\mathrm{CHCH}_{2} \mathrm{NHCH}_{2}\right), 2.69-2.57\left(10 \mathrm{H}, \mathrm{m}, 5 \mathrm{xC}_{2} \mathrm{~N}\right), 2.49\left(1 \mathrm{H}, \mathrm{tt},{ }^{2} J_{\mathrm{HP}}=23.5 \mathrm{~Hz},{ }^{3} J_{\mathrm{HH}}=5.9 \mathrm{~Hz}, \mathrm{P}_{2} \mathrm{CH}\right)$, 1.70-1.59 (4H, m, $\left.\mathrm{CH}_{2} \mathrm{CH}_{2} \mathrm{CH}_{2}\right), 1.55-1.47$ (4H, m, $\left.\mathrm{CH}_{2}\left(\mathrm{CH}_{2}\right)_{2} \mathrm{CH}_{2}\right), 1.37-1.32$ (24H, m ${ }^{17}$, $\left.\mathrm{CHMe}_{2}\right) .{ }^{13} \mathrm{C} \mathrm{NMR}\left(\mathrm{CDCl}_{3}\right): \delta \mathrm{c} 70.04\left(2 \mathrm{C}, \mathrm{m}^{17}, \mathrm{OC} H\right), 70.24\left(2 \mathrm{C}, \mathrm{m}^{17}, \mathrm{OC} H\right), 48.99$ (2C, $\left.\left.\underline{\mathrm{CH}_{2}}\left(\mathrm{CH}_{2}\right)_{2} \underline{\mathrm{CH}}_{2}\right)\right), 47.16\left(\mathrm{NH}\left(\mathrm{CH}_{2}\right)_{2} \mathrm{CH}_{2} \mathrm{NH}_{2}\right), 47.02\left(\underline{\mathrm{CH}_{2}}\left(\mathrm{CH}_{2}\right)_{2} \mathrm{NH}_{2}\right), 46.89\left(\mathrm{CHCH}_{2} \mathrm{NHCH}_{2}\right)$, $45.11\left(\mathrm{t},{ }^{2} J_{\mathrm{CP}}=4.0 \mathrm{~Hz}, \mathrm{CHCH}_{2}\right), 39.56\left(\underline{\mathrm{CH}}_{2} \mathrm{NH}_{2}\right), 37.84\left(\mathrm{t},{ }^{1} J_{\mathrm{CP}}=133.7 \mathrm{~Hz}, \mathrm{PCP}\right), 32.90$ $\left(\underline{\mathrm{CH}}_{2} \mathrm{CH}_{2} \mathrm{NH}_{2}\right), 26.98\left(\underline{\mathrm{CH}}_{2}\right), 27.01\left(\underline{\mathrm{CH}}_{2}\right), 23.20$ (4C, m $\left.{ }^{17}, \mathrm{CHMe} 2\right), 22.93\left(4 \mathrm{C}, \mathrm{m}^{17}, \mathrm{CHMe} 2\right) .{ }^{31} \mathrm{P}$ $\mathrm{NMR}\left(\mathrm{CDCl}_{3}\right): \delta_{\mathrm{P}} 21.98$.

(2-((3-((4-((3-Aminopropyl)amino)butyl)amino)propyl)amino)ethane-1,1-diyl)diphosphonic acid (6) To remove the protective groups 5 was dissolved in $4 \mathrm{M} \mathrm{HCl}(20 \mathrm{ml})$ and the mixture was refluxed for $2 \mathrm{~h}$ and concentrated under reduced pressure. Crystallization from water with ethanol gave 6 (228 mg, 70\%, purity ca. 80\%) as a brown solid. ${ }^{1} \mathrm{H}$ NMR $\left(\mathrm{D}_{2} \mathrm{O}\right): \delta_{\mathrm{H}} 3.50-3.38(2 \mathrm{H}, \mathrm{m}$, $\left.\mathrm{CHC}_{2}{ }_{2} \mathrm{NH}\right), 3.24-2.98\left(12 \mathrm{H}, \mathrm{m}, 6 \mathrm{xC}_{2} \mathrm{~N}\right), 2.56-2.42\left(1 \mathrm{H}, \mathrm{m}, \mathrm{P}_{2} \mathrm{C} \underline{\mathrm{H}}\right), 2.16-1.98(4 \mathrm{H}, \mathrm{m}$, 
$\left.\mathrm{CH}_{2} \underline{\mathrm{C}}_{2} \mathrm{CH}_{2}\right), 1.80-1.66\left(4 \mathrm{H}, \mathrm{m}, \mathrm{CH}_{2}\left(\mathrm{CH}_{2}\right)_{2} \mathrm{CH}_{2}\right) .{ }^{13} \mathrm{C} \mathrm{NMR}\left(\mathrm{D}_{2} \mathrm{O}\right): \delta \mathrm{c} 47.44\left(2 \mathrm{C}, \underline{\mathrm{CH}}_{2}\left(\mathrm{CH}_{2}\right)_{2} \underline{\mathrm{CH}}_{2}\right)$, $45.34\left(\mathrm{~m}, \mathrm{CH} \underline{C H}_{2}\right), 44.99\left(2 \mathrm{C}, \underline{\mathrm{CH}}_{2} \mathrm{NH}, \mathrm{NH} \underline{\mathrm{CH}}{ }_{2}\right), 44.77\left(\mathrm{CHCH}_{2} \mathrm{NH}^{-} \mathrm{H}_{2}\right), 37.01\left(1 \mathrm{C}, \underline{\mathrm{CH}}_{2} \mathrm{NH}_{2}\right)$, $36.43\left(\mathrm{t},{ }^{1} J_{\mathrm{CP}}=122.0 \mathrm{~Hz}\right.$ PCP $), 24.17\left(2 \mathrm{C}, \mathrm{CH}_{2} \underline{\mathrm{CH}}_{2} \mathrm{CH}_{2}\right), 23.19\left(2 \mathrm{C}, \underline{\mathrm{CH}}_{2} \underline{\mathrm{CH}_{2}}\right) .{ }^{31} \mathrm{P}$ NMR $(202.4$ $\left.\mathrm{MHz}, \mathrm{D}_{2} \mathrm{O}\right): \delta_{\mathrm{P}}$ 16.88. HRMS $[\mathrm{M}+\mathrm{H}]^{+}$calculated for $\mathrm{P}_{2} \mathrm{O}_{6} \mathrm{C}_{12} \mathrm{H}_{33} \mathrm{~N}_{4}: 391.1881$. Found: 391.1875.

\section{Labeling with technetium-99m and hydroxyapatite-binding assay}

All the bisphosphonate compounds were labeled with ${ }^{99 \mathrm{~m}} \mathrm{Tc}$ using stannous chloride reduction method. Briefly $350 \mu \mathrm{l}$ of the bisphosphonate compound ( $1 \mathrm{mg} / \mathrm{ml}$ in water), $60 \mu \mathrm{l}$ ascorbic acid $(2.5 \mathrm{mg} / \mathrm{ml}$ in saline) and $60 \mu \mathrm{l}$ stannous chloride $(1 \mathrm{mg} / \mathrm{ml}$ in saline $)$ were mixed in a glass vial and the atmosphere was replaced with nitrogen. Technetium-99m (app. $2 \mathrm{MBq}$ ) was added through the stopper. The solution was incubated at room temperature for $1 \mathrm{~h}$. After the labeling the radiochemical purity was determined by instant thin layer chromatography (ITLC) with acetone as an eluent.

The hydroxyapatite-binding assay was performed according to the procedure described by Ogawa etc. ${ }^{19}$ with some modifications. $50 \mu \mathrm{l}$ of a labeled compound and $200 \mu \mathrm{l}$ of Tris-buffered saline $(50 \mathrm{mmol} / \mathrm{l} \mathrm{pH}$ 7.5) were pipetted into microcentrifuge tube contained 1 gram of hydroxyapatite powder, the empty tube being as a control. Tubes were gently shaken at the room temperature for $30 \mathrm{~min}$ and centrifuged (15 min, $13000 \mathrm{rpm}) .50 \mu \mathrm{l}$ of the supernatant was collected and the radioactivity was measured by a gammacounter.

\section{Acknowledgements}

Authors would like to thank Mrs. Maritta Salminkoski for her skilful technical assistance. Research has been supported by the strategic funding of the University of Eastern Finland, PhoSciNet COST action CM802 and the Academy of Finland's project 132070.

\section{References}

1. Fleisch, H. Bisphosphonates in Bone Disease: From the Laboratory to the Patient, The Parthenon Publishing Group Inc.: New York, 1995.

2. Love, C.; Din, A. S.; Tomas, M. B.; Kalapparambath, T. P.; Palestro, C. J. Radiographics 2003, 23, 341 .

3. Karigiannis, G.; Papaioannou, D. Eur. J. Org. Chem. 2000, 1841.

4. Casero Jr., R. A.; Marton, L. J. Nat. Rev. Drug Discov. 2007, 6, 373.

5. Casero Jr., R. A.; Woster, P. M. J. Med. Chem. 2001, 44, 1.

6. Gordon-Weeks, R.; Parmar, S.; Davies, E.; Leigh, R. Biochem. J. 1999, 337, 373.

7. Szabo, C. M.; Oldfield, E. Biochem. Biophys. Res. Commun. 2001, 287, 468. 
8. Tokunaga, Y.; Fujisaki, J.; Takahashi, T.; Shimojo, F.; Kimura, S.; Hata, T. Proceed. Intern. Symp. Control. Rel. Bioact. Mater. 1996, 23, 615.

9. Ramakrishnan, M.; Wengenack, T.M.; Kandimalla, K.K.; Curran, G.L.; Gilles, E.J.; RamirezAlvarado, M.; Lin, J.; Garwood, M.; Jack, C.R. Jr.; Poduslo, J.F. Pharm. Res. 2008, 25, 1861.

10. Kandimalla, K.K.; Curran, G.L.; Holasek, S.S.; Gilles, E.J.; Wengenack, T.M.; RamirezAlvarado, M.; Poduslo, J.F. J Pharmacol. Exp. Ther. 2006, 318, 17.

11. Degenhardt, C. R.; Burdsall, D. C. J. Org. Chem. 1986, 51, 3488.

12. Dąbrowska, E.; Burzyńska, A.; Mucha, A.; Matczak-Jon, E.; Sawka-Dobrowolska, W.; Berlicki, L.; Kafarski, P. J. Organomet. Chem. 2009, 694, 3806

13. Chaleix, V.; Lecouvey, M. Tetrahedron Lett. 2007, 48, 703.

14. Franc, G.; Turrin, C.-O.; Cavero, E.; Costes, J.-P.; Duhayon, C.; Caminade, A.-M.; Majoral, J.-P. European J. Org. Chem. 2009, 25, 4290.

15. Turhanen, P. A.; Ahlgren, M. J.; Jarvinen, T.; Vepsalainen, J. J. Synthesis 2001, 4, 633.

16. PERCH NMR Software, 2010. Retrieved April 1, 2011 from: http://www.perchsolutions.com.

17. Vepsäläinen, J.; Nupponen, H.; Pohjala, E. Tetrahedron Lett. 1993, 34, 4551.

18. Vepsäläinen, J.; Nupponen H.; Pohjala E.; Ahlgren M.; Vainiotalo P. J. Chem. Soc., Perkin Trans. 2 1992, 835.

19. Ogawa, K.; Mukai, T.; Inoue, Y.; Ono, M.; Saji, H. J. Nucl. Med. 2006, 47, 2042.

20. Nancollas, G. H.; Tang, R.; Phipps, R. J.; Henneman, Z.; Gulde, S.; Wu, W.; Mangood, A.; Russell, R. G. G.; Ebetino, F. H. Bone, 2006, 38, 617. 